\title{
In-Situ and Ex-Situ Fe K-Edge X-Ray Absorption Spectroscopic Studies for the Structural and Electronic Evolution of Strontium Ferrite upon Electrochemical Oxidation
}

\author{
J.-H. Choy, D.-H. Kim and S.-H. Hwang \\ Center for Molecular Catalysis, Department of Chemistry, College of Natural Sciences, Seoul National \\ University, Seoul 151-742, Korea
}

\begin{abstract}
A structural evolution from $\mathrm{SrFeO}_{2.5}$ (brownmilierite) to $\mathrm{SrFeO}_{3}$ (perovskite) upon electrochemical oxidation has been systematically studied by in-situ and ex-situ X-ray Diffraction(XRD) and X-ray absorption spectroscopy(XAS). According to the XRD analysis, the orthorhombic $\mathrm{SrFeO}_{2.5}$ has been gradualiy converted to the cubic $\mathrm{SrFeO}_{3}$ as the reaction proceeds. The mostly oxidized perovskite could be been obtained after $66 \mathrm{~h}$, From the in-situ and ex-situ Fe K-edge XANES study, it has been found that the degree of oxidation goes to a maximum after $48 \mathrm{~h}$ and $30 \mathrm{~h}$ for the bulk and the surface, respectively. EXAFS results are also consistent with the above findings from the fact that no peak evolution could be observed after $48 \mathrm{~h}$ and $30 \mathrm{~h}$ for both ex-situ and in-situ studies, respectively.
\end{abstract}

\section{INTRODUCTION}

Thanks to its simple and optically-opened instrumentation, the electrochemical oxidation[1-3] can be combined with various spectroscopic methods. Among them, XAS measurement is known to be a powerful methodology to investigate the evolution of electronic and local structures of the material during the reaction[3-5]. Here, we report the in-situ $\mathrm{XAS}_{\text {results of }} \mathrm{SrFeO}_{3}$, of which $\mathrm{Fe}^{3+}$ ion converts completely to $\mathrm{Fe}^{4+}$ one due to the electrochemical oxidation. Powder XRD and ex-situ XAS studies also have been performed to compare with the in-situ XAS results.

\section{EXPERIMENTAL}

Starting material, $\mathrm{SrFeO}_{2.5}$ has been prepared by decomposing nitrate precursors at $1200^{\circ} \mathrm{C}$ in air, followed by annealing in $\mathrm{N}_{2}$ atmosphere at $1000^{\circ} \mathrm{C}$. Electrochemical oxidation has been performed by potentiostatic method, applying $+400 \mathrm{mV}$ (vs. $\mathrm{Hg} / \mathrm{HgO}$ ) potential to a $\mathrm{RDE}$ type working electrode $\left(\mathrm{SrFeO}_{2.5}\right.$ pellet). Powder XRD patterns have been obtained from a Phillips PW1830 diffractometer using Ni-filtered $\mathrm{Cu} \mathrm{K}_{\alpha}$ radiation. XAS measurements have been carried out with synchrotron radiation by using the EXAFS facilities installed at beam line $7 \mathrm{C}$ of the Photon Factory, Tsukuba, operated at $2.5 \mathrm{GeV}$ with
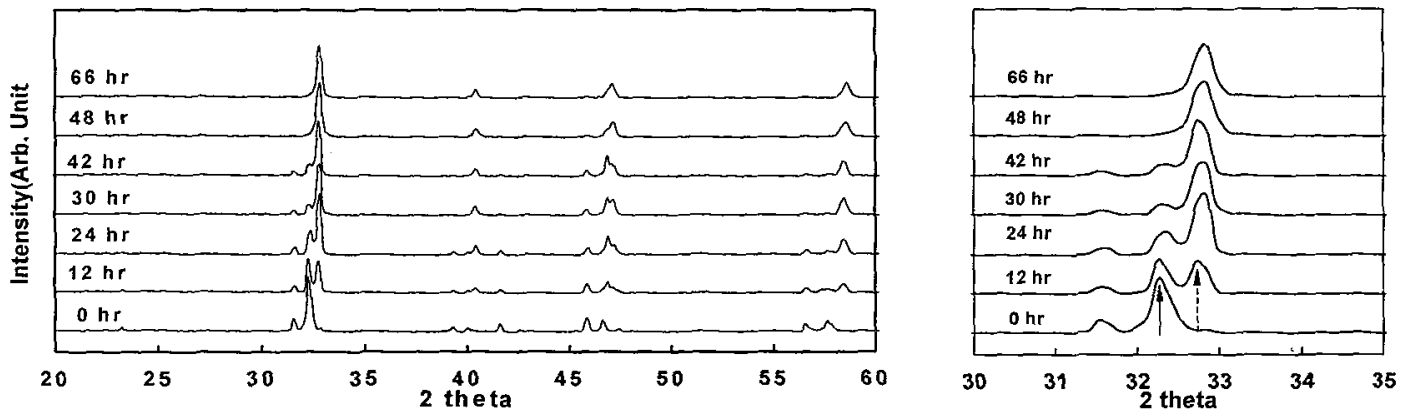

Figure 1. Powder $\mathrm{X}$-ray Diffraction patterns of electrochemically oxidized $\mathrm{SrFeO}_{3}$ as a function of reaction time. Solid and broken arrows in the right pattern indicate the brownmillerite (141) reflection and perovskite (110) reflection, respectively. 

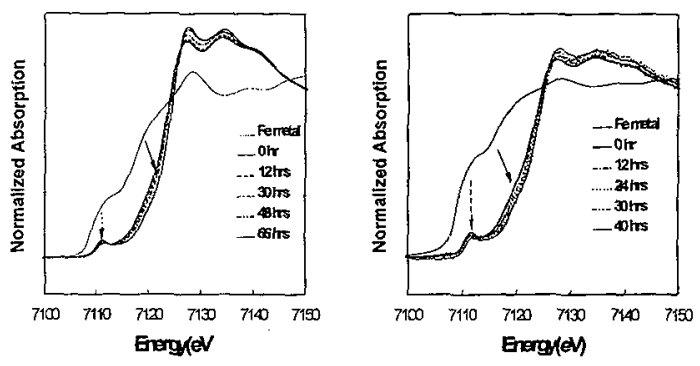

Figure 2. Time-dependent ex-situ(left) and in-situ(right) Fe K-edge XANES spectra of $\mathrm{SrFeO}$.
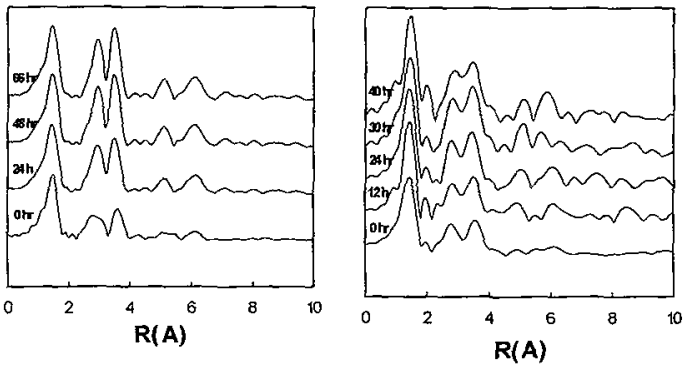

Figure 3. The Fourier transform of the time-dependent exsitu(left) and in-situ(right) $\mathrm{Fe}$ K-edge EXAFS data for $\mathrm{SrFeO}_{3}$.

about 260-360 mA of stored current. Samples for ex-situ measurements were prepared by pelleting BN:sample mixture(10:1 w/w). In-situ measurements were performed by using Lytle type fluorescence detector and Teflon reaction cell with Kepton window at the front panel.

\section{RESULTS AND DISCUSSION}

Time-dependent powder XRD patterns(Fig. 1) show gradual formation of cubic phase along the reaction time. Significant degradation of orthorhombic symmetry can be observed even after $12 \mathrm{~h}$, and after $66 \mathrm{~h}$, all the main peaks can be indexed upon cubic perovskite unit cell except a small one at $46^{\circ}$ corresponding to an orthorhombic phase, whose intensity is negliglbly smail(less than $5 \%$ ) compared to the main peak, indicating that all the oxygen vacancy is almost filled by electrochemical oxidation.

In-situ and ex-situ Fe K-edge XANES measurements(Fig. 2) gave similar results. The time-dependent, continuous increase of absorption edge energy(indicated by solid arrow in the figure) and decreases of pre-edge(broken arrow) peak intensity show the stabilization of $\mathrm{Fe}^{4+}$ and the increase of octahedral sites in the lattice along the oxidation. The spectra corresponding to 48 $\mathrm{h}$ and $66 \mathrm{~h}$ for the ex-situ measurement give no significant difference in shape, pre-edge intensity, and absorption energy. Insitu measurements of the samples oxidized for $30 \mathrm{~h}$ and $40 \mathrm{~h}$ show the same results as the ex-situ case. From the above observation, it can be concluded that the bulk and surface oxidation of $\mathrm{SrFeO}_{3}$ can be achieved slightly more than $48 \mathrm{~h}$ and 30 $h$, respectively.

Analyses of the in-situ and ex-situ Fe K-edge EXAFS spectra also support the XRD and XANES results. From Fig. 3, the time-dependent evolution of Fourier-transformed spectra given in $\mathrm{R}$ space represents the continuous oxidation which comes to the maximum around $48 \mathrm{~h}$ (bulk) and $30 \mathrm{~h}$ (surface). In addition, the $\mathrm{Fe}-\mathrm{O}$ distance of mostly oxidized sample obtained from EXAFS fitting is $1.91 \AA$, which is well consistent with the crystallographic data(1.93 $\AA$ ) in the error limit of EXAFS fitting. The increment of peak intensities around $3.8 \AA(\mathrm{Fe}-\mathrm{O}-\mathrm{Fe})$ and $5.4 \AA$ ( $\mathrm{Fe}-\mathrm{Fe}$ ) along the reaction time indicate the increase of octahedral site as the oxidation proceeds. Fittings based on the model of mixed crystal symmetry(orthorhombic and cubic) are now on the way, and will give the more precise understandings for this system.

\section{SUMMARY}

The structural transformation from brownmillerite $\left(\mathrm{SrFeO}_{2.5}\right)$ to perovskite $\left(\mathrm{SrFeO}_{3}\right)$ has been electrochemically made and its insitu observation has been also performed XRD and XAS studies. According to the in-situ XAS results, it is understood that the sample surface is completely oxidized after $30 \mathrm{~h}$, while the bulk needs at least $48 \mathrm{~h}$ to form perovskite with minor oxygen vacancy. The stabilization of $\mathrm{Fe}^{\phi+}$ in the crystal lattice and the existence of distorted local symmetry have been confirmed by XANES study. Based upon the good consistency between in-situ XAS results and ex-situ one, it is found that XAS spectroscopic method is a powerful tool to elucidate the variation of the electronic and the local structure of the material during the electrochemical reaction.

\section{Acknowledgments}

This work was supported in part by non-directed research fund, Korea Research Foundation.

\section{References}

[1] Wattiaux A., Fournes L., Demourgues A., Bernaben N., Grenier J.-C., and Pouchard M., Solid State Comm 77 (1991) $489-$ 493

[2] Choy J.H., Park J.C., Kang S.G., Kim D.H., and Lee J.Y., Synth. Metals 71 (1995) 1627-1628

[3] Choy J.H., Kim D.K., Hwang S.H., and Park J.C., J. Am. Chem. Soc. 117 (1995) 7556-7557

[4] Choy J.H., Kim. D.K., Hwang S.H., and Demazeau G., Phys. Rev. B50 (1994) 16631-16639

[5] Choy J.H., Kim. D.K., Hwang S.H., Demazeau G., and Jung D.Y., J. Am. Chem. Soc. 117 (1995) $8557-8566$ 